\title{
MEDICAL SCHEMES OVERCOMING HIV/AIDS HURDLES
}

The low registration of patients on HIV management programmes has received a fair amount of negative publicity recently. However, research suggests that enrolment of those who stand to gain the most benefit from accessing treatment is relatively high. In addition, the inclusion of antiretroviral therapy into the HIV prescribed minimum benefits (PMBs) will have a limited effect on medical schemes.

This is according to Rodney Cowlin, managing director for Aid for AIDS, a Medscheme initiative that has been in operation for almost seven years. 'Aid for AIDS has looked critically at number of beneficiaries registered on HIV programmes in relation to the estimated HIV prevalence within medical schemes', he says.

To ensure accuracy, the analysis was carried out on several medical schemes covering 680000 lives and the HIV prevalence for these schemes was calculated to be $10 \%$ as of early 2005.

'To date, $2.7 \%$ of beneficiaries from these schemes are enrolled on AfA (1.9\% are receiving antiretroviral therapy). This includes people at all stages of the disease,' says Cowlin. 'While this enrolment may sound low, it is unlikely that all HIV-positive beneficiaries would enrol on an HIV management programme, either because the disease is in its early stages, and they have no or few symptoms and are unaware of their HIV status, or because of the perception that there is no reason to register on HIV management programmes until antiretroviral therapy is required.

An alternative approach to analysing statistics would be to look at the number of HIV-positive beneficiaries who have joined HIV management programmes as a percentage of the number that should be on antiretroviral therapy (ART). 'Based on data from the World Health Organization (WHO), we estimate that at least 21\% of HIV-positive patients should be on ART. Using the 10\% prevalence indicated by the modelling exercise, this equates to $2.1 \%$ of the 680000 lives. Currently, 1.9\% of lives are actually on ART, which is close to the estimated number who should be on treatment', Cowlin says.
The data therefore suggest that enrolment of those who stand to gain most benefit from registering on AfA and accessing treatment is relatively high. 'However, this does not mean that management programmes should not continue to make every effort to enrol as many HIVpositive beneficiaries as possible. Early enrolment provides a valuable educational opportunity and enables ART to be commenced at the optimal time.'

\section{INCLUSION OF ART INTO THE HIV PMBs}

From 1 January 2005 the PMBs related to HIV infection have include the provision of ART and appropriate monitoring tests. However, a 2002 report from the Centre for Actuarial Research, University of Cape Town showed that 90\% of medical aid beneficiaries already had access to highly active antiretroviral therapy (HAART). 'Currently all 32 of the medical schemes contracted to AfA make provision for HAART across all benefit options.' As a result, the new regulations are likely to have little impact on the industry as a whole. The provision of HAART will in fact improve the financial position of those schemes that did limit access to HAART in the past, as a result of the favourable impact on hospital admissions. Further benefit is possible if the scheme makes use of an HIV management programme. 'Analysis of HIV-related expenditure incurred by the schemes referred to in the AfA modelling exercise shows that the monthly cost of treating a patient who requires ART (first-line therapy) is R935.00. This includes ART, consultations, monitoring tests, preventive antibiotics and the management of common associated conditions', Cowlin says.

Generally speaking, medium- to high-contribution medical scheme options offer good benefits of up to R25 000 per beneficiary per annum, which is more than adequate to cover treatment costs for a year (HAART, including second-line and salvage therapy, PEP and PMTCT, related medicines and pathology tests).

'Furthermore', says Cowlin, 'anticipated reductions in the cost of drugs and monitoring tests will assist schemes to continue providing reasonable benefits for the treatment of HIV/AIDS.' 